\title{
A HYDRODYNAMICAL MODEL FOR THE FRAGMENTATION OF THE W49A STAR-FORMING REGION*
}

\author{
JOHN LATTANZIO ${ }^{1,2}$, ERIC KETO ${ }^{2}$ and JOE MONAGHAN ${ }^{1}$ \\ ${ }^{1}$ Department of Mathematics, Monash University, \\ Clayton, Victoria, 3168, AUSTRALIA. \\ ${ }^{2}$ Institute of Geophysics and Planetary Physics, L-413, \\ L.L.N.L., P.O. Box 808, Livermore, Ca., 94550, USA.
}

\begin{abstract}
We present a 3-D hydrodynamical and radiative transfer simulation which suggests that the circular "necklace" of massive star formation in W49A may result from fragmentation via a ring instability during the collapse of a rotating cooling molecular cloud.
\end{abstract}

\section{Introduction}

The star-forming region $\mathrm{W} 49 \mathrm{~A}$ is characterized by a rotating ring of HII regions (Welch et al. 1987). Spectral lines toward several of the HII regions show red and blue shifted absorption and emission components split in an inverse P-Cygni profile characteristic of infall and gravitational collapse (Keto 1990a). Welch et al. interpret this as evidence for uniform collapse of a several pc scale molecular envelope onto the ring. They postulate a central mass of $5 \times 10^{4} M_{\odot}$ to keep the orbiting HII regions in centrifugal equilibrium, and motivate the infall of the cloud envelope. We present here an alternative model.

\section{The Calculations}

We begin with the cooling spherical cloud described in Monaghan and Lattanzio (this volume, and 1990). The gas rapidly collapses down the rotation axis and forms a thin disc. After 2 initial free fall times $\left(t_{f f}\right)$ a ring forms, with material in the centre cleared due to the centrifugal force. The ring then fragments into 5 major condensations (see figure 1 ). These cores have masses of about $1000 M_{\odot}$ and radii about $0.6 \mathrm{pc}$.

We expect stars to form within these cores, on a scale below our resolution. We have thus assumed that in each core there is an HII region of diameter $0.1 \mathrm{pc}$. The model HII

* Work performed under the auspices of the U.S. Department of Energy by the Lawrence Livermore National Laboratory under contract No. W-7405-ENG-48. 
regions serve 2 purposes. They heat the surrounding molecular gas (Scoville and Kwan, 1976) and provide a background source of continuum radiation against which the foreground gas may be seen in absorption. Synthetic $\mathrm{HCO}^{+}$spectra calculated with the code described by Keto (1990b) are shown in figure 1 . The velocity field in the model shows that the splitting of the emission and absorption lines arises from small scale rotation and collapse within the individual cores. The magnitude of the splitting is about the same in each of the different cores because the fragmentation process results in cores of approximately equal mass and radius. Whether the splitting is seen in emission or absorption is dependent on the assumed radiation temperature of the continuum relative to the lines.

\section{The New Model}

In this model we propose that the ring of HII regions results from the fragmentation, through a ring-mode, of a collapsing rotating cloud of gas. There is no central mass to keep the fragments in stable orbits, and further hydrodynamical evolution shows the ring to be a transient phenomenon with a lifetime of $<t_{f s}$. Gravitational collapse at the time of formation of the cores is entirely localized about the individual HII regions while the surrounding disc material is only weakly bound to the structure. Details will be published elsewhere (Keto et al. 1990).
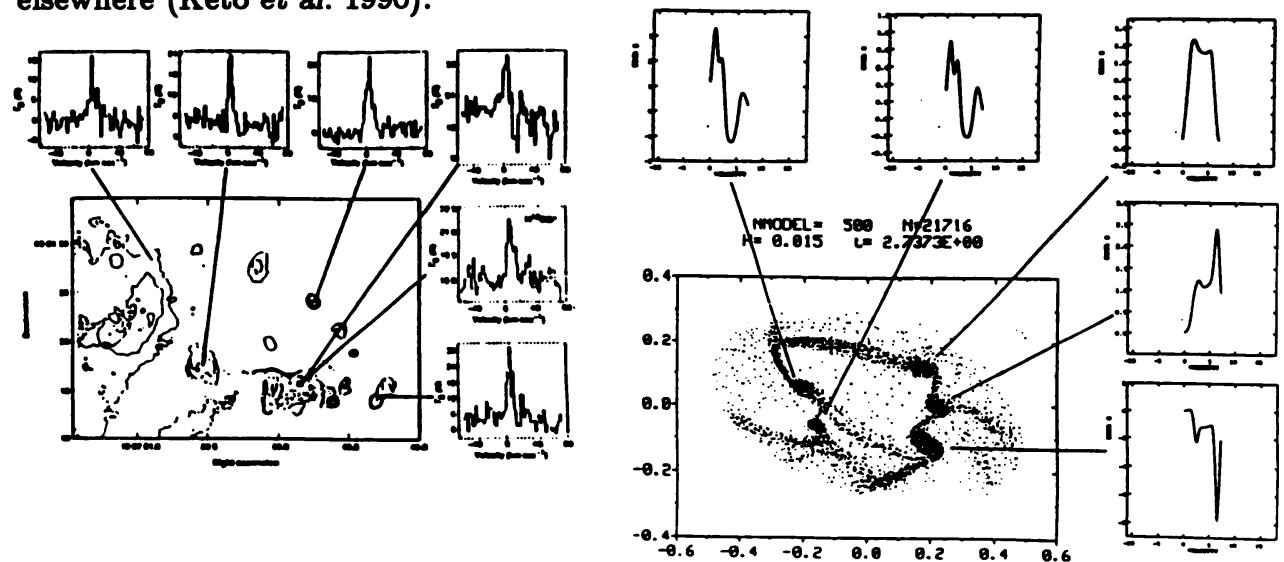

Figure 1: W49A (left) from Welch et al. (1987) and our model (right), at a viewing perspective suggestive of $\mathrm{W} 49 \mathrm{~A}$.

\section{References}

Keto, E. R., 1990a, Astrophys. J., 355, 190.

Keto, E. R., 1990b, Astrophys. J., in press.

Keto, E. R., Lattanzio, J. C., and Monaghan, J. J., 1990, in preparation.

Monaghan, J. J., and Lattanzio, J. C., 1990, Astrophys. J., submitted.

Scoville, N., and Kwan, J., 1976, Astrophys. J., 206, 718.

Welch, W. J., Dreher, J. W., Jackson, J. M., Tereby, S., and Vogel, S. N., 1987, Science, 238, 1550. 